\title{
Validation of the Publication of New Names and New Combinations Previously Effectively Published Outside the IJSB
}

\author{
List No. $63 \dagger$
}

The purpose of this announcement is to effect the valid publication of the following new names and new combinations under the procedure described previously [Int. J. Syst. Bacteriol. 27(3):iv, 1977]. Authors and other individuals wishing to have new names and/or combinations included in future lists should send the pertinent reprint or a photocopy thereof to the IJSB (c/o ASM) for confirmation that all of the other requirements for valid publication have been met. It should be noted that the date of valid publication of these new names and combinations is the date of publication of this list, not the date of the original publication of the names and combinations. The authors of the new names and combinations are as given below, and these authors' names will be included in the author index of the present issue and in the volume author index in this issue of the IJSB.

Inclusion of a name on these lists validates the name and thereby makes it available in bacteriological nomenclature. The inclusion of a name on this list is not to be construed as taxonomic acceptance of the taxon to which the name is applied. Indeed, some of these names may, in time, be shown to be synonyms, or the organisms may be transferred to another genus, thus necessitating the creation of a new combination.

\begin{tabular}{|c|c|c|c|c|}
\hline Name & Proposed as: & Author(s) (reference) & Priority $^{a}$ & Nomenclatural type $^{b}$ \\
\hline Borrelia tanukii & New species & Fukunaga et al. (3) & 3 & Strain Hk501 (= JCM 9662) \\
\hline Borrelia turdi corrig. (Borrelia turdae $[\mathrm{sic}])^{c}$ & New species & Fukunaga et al. (3) & 3 & Strain Ya501 (= JCM 9661) \\
\hline Caldicellulosiruptor lactoaceticus & New species & Mladenovska et al. (6) & 6 & Strain 6A (= DSM 9545) \\
\hline Corynebacterium imitans & New species & Funke et al. (4) & 7 & $\begin{array}{l}\text { NCTC } 13015(=\text { CCUG } 36877= \\
\quad \text { DSM 44264) }\end{array}$ \\
\hline Corynebacterium lipophiloflavum & New species & Funke et al. (5) & 4 & $\begin{array}{l}\text { Strain DMMZ } 1944 \text { (= CCUG } 37336 \\
\quad=\text { DSM 44291) }\end{array}$ \\
\hline Desulforhabdus & New genus & Oude Elferink et al. (7) & 2 & Desulforhabdus amnigenus \\
\hline Desulforhabdus amnigenus & New species & Oude Elferink et al. (7) & 2 & $\begin{array}{l}\text { Strain ASRB1 (= ATCC } 51979= \\
\quad \text { DSM 10338) }\end{array}$ \\
\hline Desulfospira & New genus & Finster et al. (2) & 5 & Desulfospira joergensenii \\
\hline Desulfospira joergensenii & New species & Finster et al. (2) & 5 & Strain B331 (= DSM 10085) \\
\hline Halobacteroides elegans & New species & Zhilina et al. (8) & 1 & Strain Z-7287 (= DSM 6639) \\
\hline Psychrobacter glacincola & New species & Bowman et al. (1) & 5 & ACAM 483 \\
\hline
\end{tabular}

$\uparrow$ Lists 1 through 62 were published in the Int. J. Syst. Bacteriol. 27:306, 1977; 29:79, 436, 1979; 30:601, 676, 1980; 31:215, 382, 1981; 32:266, 384, 1982; 33:438, 672, 896,$1983 ; \mathbf{3 4}: 91,270,355,503,1984 ; \mathbf{3 5}: 223,375,535,1985 ; \mathbf{3 6}: 354,489,573,1986 ; \mathbf{3 7}: 179,1987 ; \mathbf{3 8}: 136,220,328,449,1988 ; \mathbf{3 9}: 93,205,371,495,1989 ; \mathbf{4 0}: 105,212$,

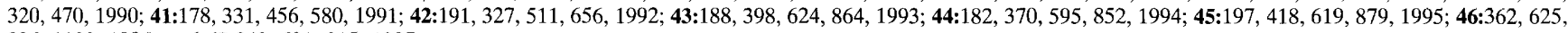
$836,1189,1996$; and 47:242, 601, 915, 1997.

${ }^{a}$ Priority number assigned according to the date the documentation and request for validation are received.

${ }^{b}$ Abbreviations: ACAM, Australian Collection of Antarctic Microorganisms, Hobart, Tasmania, Australia; ATCC, American Type Culture Collection, Rockville, Md.; CCUG, Culture Collection, University of Göteborg, Göteborg, Sweden; DSM, DSMZ-Deutsche Sammlung von Mikroorganismen und Zellkulturen GmbH, Braunschweig, Germany; JCM, Japan Collection of Microorganisms, Riken, Saitama, Japan; NCTC, National Collection of Type Cultures, London, England.

${ }^{c}$ Name has been corrected in accordance with Rule 61 of the Bacteriological Code on the occasion of validation.

\section{REFERENCES}

1. Bowman, J. P., D. S. Nichols, and T. A. McMeekin. 1997. Psychrobacter glacincola sp. nov., a halotolerant, psychrophilic bacterium isolated from Antarctic sea ice. Syst. Appl. Microbiol, 20:209-215.

2. Finster, K., W. Liesack, and B. J. Tindall. 1997. Desulfospira joergensenii, gen. nov., sp. nov., a new sulfate-reducing bacterium isolated from marine surface sediment. Syst. Appl. Microbiol. 20:201-208.

3. Fukunaga, M., A. Hamase, K. Okada, and M. Nakao. 1996. Borrelia tanukii sp. nov. and Borrelia turdae sp. nov. found from ixodid ticks in Japan: rapid species identification by $16 \mathrm{~S}$ rRNA gene-targeted PCR analysis. Microbiol. Immunol. 40:877-881.

4. Funke, G., A. Efstratiou, D. Kuklinska, R. A. Hutson, A. de Zoysa, K. H. Engler, and M. D. Collins. 1997. Corynebacterium imitans sp. nov. isolated from patients with suspected diphtheria. J. Clin. Microbiol. 35:1978-1983.
5. Funke, G., R. A. Hutson, M. Hilleringmann, W. R. Heizmann, and M. D. Collins. 1997. Corynebacterium lipophiloflavum sp. nov. isolated from a patient with bacterial vaginosis. FEMS Microbiol. Lett. 150:219-224.

6. Mladenovska, Z., I. M. Mathrani, and B. K. Ahring. 1995. Isolation and characterization of Caldicellulosiruptor lactoaceticus sp. nov., an extremely thermophilic, cellulolytic, anaerobic bacterium. Arch. Microbiol. 163:223230

7. Oude Elferink, S. J. W. H., R. N. Maas, H. J. M. Harmsen, and A. J. M. Stams. 1995. Desulforhabdus amnigenus gen. nov. sp. nov., a sulfate reducer isolated from anaerobic granular sludge. Arch. Microbiol. 164:119-124.

8. Zhilina, T. N., T. P. Turova, A. M. Lysenko, and V. V. Kevbrin. 1997. Reclassification of Halobacteroides halobius Z-7287 on the basis of phylogenetic analysis as a new species Halobacteroides elegans sp. nov. Mikrobiologiya 66:114-121. 